\title{
Multivariate statistics and three-dimensional graphics
}

\author{
MICHAEL YOST and LAWRENCE GINDLER \\ Trinity University, San Antonio, Texas
}

and

\author{
FREDERICK J. BREMNER \\ Brooks Air Force Base School of Aerospace Medicine, San Antonio, Texas \\ and Trinity University, San Antonio, Texas
}

\begin{abstract}
This paper describes the process by which a system of three-dimensional computer-generated graphics was developed for use by students and researchers in analyzing multivariate psychological data. Examples of the application and use of the system in analyzing factor analytic and discriminant analysis data are presented. Details on the operation of the system are also included.
\end{abstract}

Multivariate statistics contain a variety of descriptive and inferential techniques that have been developed to handle cxperimental situations in which sets of variables are involved either as predictors or as measures of performance. If psychologists were more narrow-minded, or psychological theories and measures were not so well developed, or nature were so simple as to dictate a single independent variable and a single outcome measure in each study, there would be no need for multivariate statistical techniques.

The classic psychological experiment involves a single manipulated variable and a single outcome measure, and all other variables are eliminated as casual factors either through explicit experimental control or through statistical control. Questions of patterns of optional combinations of variables seldom arise, and the problems of multiple comparisons do not get in the way of interpretations of $t$ tests or correlation coefficients. Researchers in psychology and the other social and behavioral sciences have moved away from sole reliance on classic, univariate experimental and statistical designs. Over the years, psychologists have moved toward agreement that a given experimental manipulation can affect many somewhat different, but partially correlated, aspects of a subject's behavior. Similarly, many different pieces of information about an applicant for admissions or employment may be of value in predicting his/her academic success in school or performance on the job.

The commonly employed techniques in multivariate statistics are straightforward generalizations of a univariate statistical tool, with a single variable being replaced by a linear combination of several original variables. The complex algebra and calculus that make multivariate statistics seem so inaccessible is simply a substitute for a trial-

Address correspondence to Michael Yost, Office of the President, Trinity University, 715 Stadium Dr., San Antonio, TX 78284. and-error selection of weights for variables entering into a linear combination so as to achieve the best fit to some criteria.

When, in the 1930s, Kendall, Mahalanobis, and others derived some of their original multivariate equations and tests of significance (Kendall, 1969), it took many manhours to complete the calculations for even a simple experiment. In the 1960 s, when computers were proliferating, several general-purpose multivariate statistical programs were developed (Clyde, 1969; Finn, 1965; Hughes, LaRue, \& Yost, 1969). Most of these programs required the use of large computers, which were available to only a limited number of individuals. Almost no undergraduate students had access to multivariate statistics on computers. Throughout the 1970 s, mainframes increased in number, the number of general-purpose multivariate statistical computer programs increased, and students at the master's and doctoral levels gained more access to multivariate statistical techniques. In these years faculty and graduate students spent more time waiting for output from the computer then they did analyzing data. The proliferation of microcomputers began in the early 1980s and continues, and the development of worthwhile multivariate computer programs for use on these computers began in the mid-1980s. Now, multivariate statistical techniques can be used by almost any investigator or student. Given the current availability of microcomputers and multivariate computer programs, there is very little reason for a person who has a need for multivariate statistics not to use them.

The statistical solution to a simple, two-group univariate hypothesis usually involves the interpretation of two numbers (as in a $t$ test, where an investigator interprets the $t$ value and the probability statement), the means and standard deviations of the scores, and the shapes of the distributions of the scores. Although the calculations of the statistics (both descriptive and inferential) are al- 
gebraic, the interpretations that are drawn or inferences that are made are largely geometric in nature. The distribution of scores and their interpretation in univariate statistical analyses are two-dimensional. This makes the development and interpretation of this type of hypothesis relatively straightforward.

The testing of a multivariate hypothesis involves solving a number of complex equations. The final solution to multivariate analyses is typically a series of values that are used in conjunction with a number of other vectors or matrices. Although the solution and interpretation of univariate problems is basically a two-dimensional problem, the solution and interpretation of multivariate problems (discriminant analysis, factor analysis, canonical solutions, etc.) is $K$-dimensional (where $K$ is either the number of variables or the number of solutions). $\mathrm{Ob}$ viously, it is impossible to visually display anything more than a three-dimensional space; however, it is critical that an investigator or student be able to conceptualize and work in a three-dimensional space.

At Trinity University, we teach basic statistical techniques to both our graduate and our undergraduate students. We have used a number of multivariate statistical packages and, except for some of the idiosyncrasies of the programs, have had no real problems. The main problem is that it is difficult for individuals who are new to multivariate statistics to conceptualize and work with a space of three or more dimensions. Although programs such as SPSS (1985) and SAS (1986) can print combinations of all two-dimensional graphs of a three-dimensional space, these graphs do not always accurately describe the phenomena being studied. In fact, the production and interpretation of many of these graphs is frequently confusing. We move to the use of multivariate statistics because of the complexity of a study and then, when the study has been completed, we retreat to combinations of univariate statistics because we cannot conceptualize the complex solution.

\section{The System}

For the last 12 years, we have been working to develop computerized tools that would permit the development of a graph of three-dimensional space on a computer screen. Furthermore, we have been trying to develop a system that would permit us, as well as our students in statistics classes, to identify the data of different groups of subjects on the screen and to be able to rotate this data display around any of its three axes $(x, y, z)$. Our initial efforts were to develop this system on a mainframe computer. Although we had some success in developing this system, it proved to be cumbersome to use, it was not available for many users, data were difficult to enter, and the system consumed a large part of the capacity of the mainframe computer. We now have developed a microcomputer-based system that is based on commercially available software that should satisfy laboratory needs and the tutorial needs of students.

The system is based on a number of multivariate statistical programs and two programs for Macintosh computers.
SAS (1986), SPSS (1985), and Hughes et al.'s (1969) program are used to analyze data, and the traditional output for the solution is obtained. This output includes the weighted linear composite scores for each subject for each factor. The raw data for each subject and the composite scores are edited into a single matrix. In a multisubject design, the data are in a variables (columns) $\times$ subjects (rows) matrix with factors and levels represented as variables. In a single-subject design, the data are in a variables (columns) $\times$ trials (rows) matrix with factors and levels represented as variables. The data matrix used with the multivariate statistical program is copied to a floppy disk and transferred to the microcomputer laboratory. Our microcomputer lab is equipped with Macintosh SEs, which have $1 \mathrm{MB}$ of memory and a 9-in. screen with $512 \times 342$ pixels. In the event that the data are brought from an IBM PC, mainframe, or other computer, they can be made Macintosh compatible by using the Apple file-exchange program or other PC-to-Macintosh filetransfer utilities. The data matrix is first read into Excel or another spreadsheet program for editing and is then placed into MacSpin (Donoho, Donoho, \& Gasko, 1986), which is a three-dimensional graphics computer program. We maintain a library of floppy disks on which data matrices are stored for different studies. These cataloged matrices are used for classroom instruction, student tutorials, and student projects.

MacSpin contains a very powerful and easily used data editor. With this editor, it is easy to composite variables, to make linear or curvilinear transformations, or to make standard score transformations. This editor is more powerful and easily used than the editor in the multivariate statistical program.

In the section that follows, we describe the use of threedimensional graphics with psychological data. We begin with factor analysis and move to a single-factor multilevel discriminant analysis (multivariate analysis of variance, or MANOVA). Multiple correlation or regression is not included. Since the geometric explanation of this statistic requires only the relationship of $Y$ to $\hat{Y}$, this is a twodimensional problem and the solution is available with many existing computer programs.

\section{Factor Analysis}

The term factor analysis refers to a wide variety of statistical techniques. All of the techniques have in common the explicit separation of unique variance from common variance, and they assume that the intercorrelations among the original variables are generated by a number of latent variables. The object of most of these techniques is to identify, explain, and label latent variables through an analysis of the original variables. Although the mathematical explanation of the basic approach to factor analysis is fairly complex, the geometric explanation is straightforward. Imagine that there is a set of $N$ trivariate (three variables per observation) observations and that these observations can be displayed as a scatterplot. Figure 1 is a three-dimensional scatterplot in which the $x$, $y$, and $z$ axes represent scores on the Scholastic Aptitude 


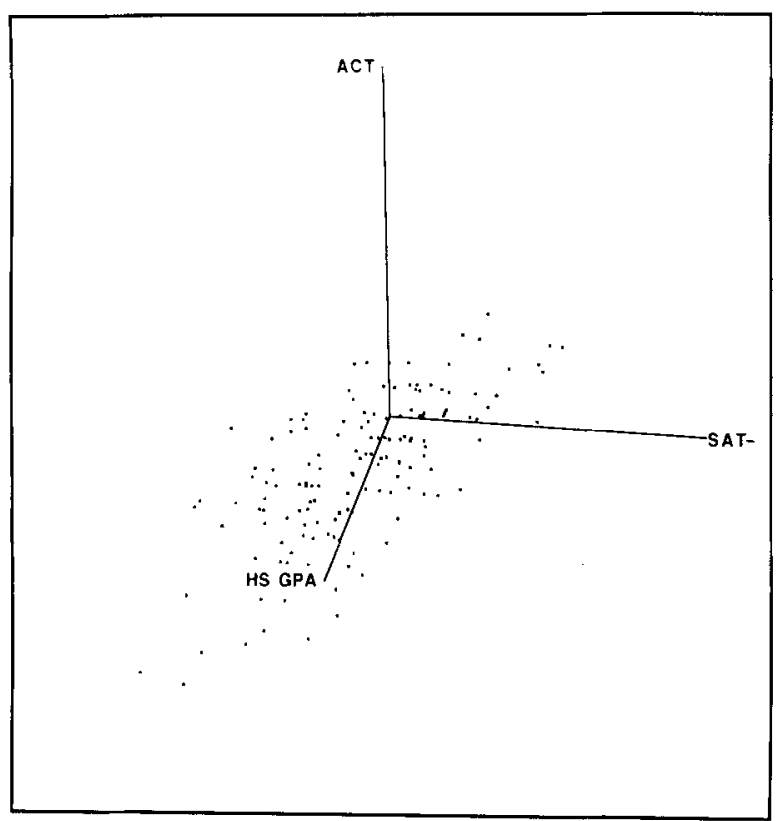

Figure 1. A swarm of data representing prospective college students' scores on the Scholastic Aptitude Test (SAT), scores on the American College Test (ACT), and high school grade point averages (HS GPA). The axes have been rotated to show maximum length of the swarm $\left(9^{\circ}\right.$ latitude and $16^{\circ}$ longitude).

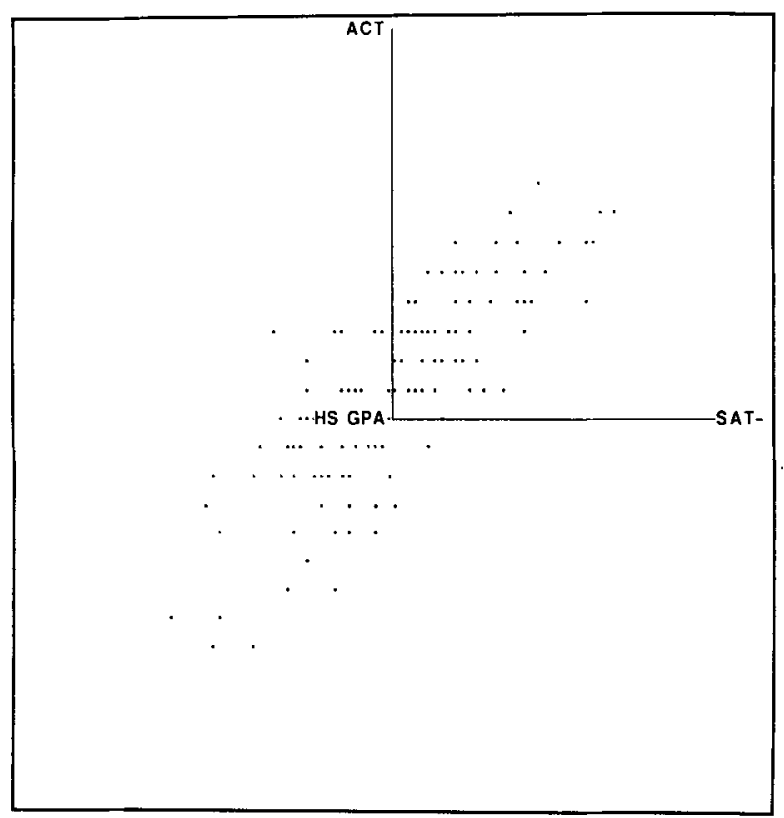

Figure 2. The same set of scores presented in Figure 1, but viewed from the end of the HS GPA axes. Note the decrease in width of the swarm.

Test (SAT), scores on the American College Test (ACT), and high school grade point averages (HSGPA), respectively. Since a factor analysis has its basis in correlation, each of the variables is in a standard score format, and the means of the scores are at the intersection of the three axes. Note that the swarm of data points has a general three-dimensional ellipsoid shape. The ellipsoid swarm appears to have a major axis that lies somewhere between the three original axes. This axis is known as the first factor or first principal component of the solution. Unlike the three original axes, which were univariate axes, this new axis (the first factor) is multivariate and is a weighted linear composite of the three original variables. For interpretation purposes, the weighted contribution of each of the original variables to the first factor is actually the projection of the first factor on each of the axes of the three original variables. The larger the projection on an axis (i.e., the larger the weighted contribution that variable makes to the composite), the larger the contribution of the variable to the interpretation and naming of the factor.

There is a second axis within the original ellipsoid swarm that is perpendicular to the first. This one is obviously much shorter than the first, and it is the second factor to the solution. Since this factor is, by definition, perpendicular to the first, the information defined by this factor is independent of the information defined by the first factor. The relationship between the second factor and the original variables is identical to the relationship between the first factor and the original variables. Since it is possible (but seldom desirable) to have as many factors as there are variables, the third factor (and all succeeding factors) is interpreted in the same way as the first two.

The eigenvalues, factor loadings, communalities, and factor weights are mathematical indicators used to interpret factor analyses. Basically, these numeric values describe the geometric relationships within the $K$-dimensional (where $K$ is the number of variables) space. In a teaching situation, students do not find it easy to understand the relationship between the mathematics and the geometric

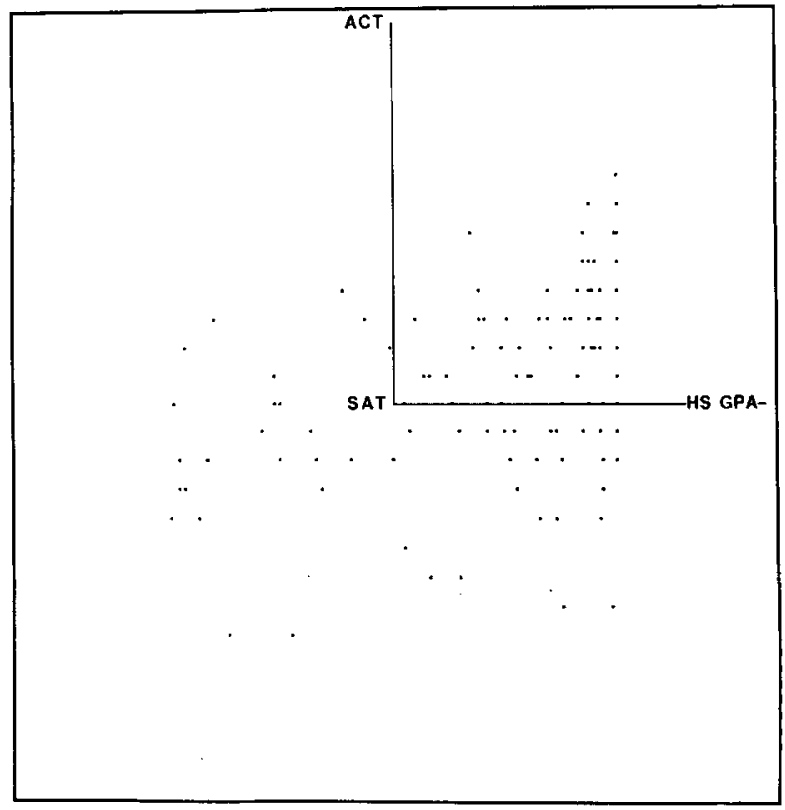

Figure 3. The same set of scores presented in Figure 1, but viewed from the end of the SAT axes. Note the increase in width of the swarm. 
Table 1

Discriminant Analysis

\begin{tabular}{lrrrrrr}
\hline & \multicolumn{2}{c}{ Males } & & \multicolumn{2}{c}{ Females } & \\
\cline { 2 - 3 } \cline { 5 - 6 } & Males & $S D$ & & Fermales & $S D$ & Correlations* \\
\hline SAT & 1240 & 105 & & 1180 & 110 & .85 \\
ACT & 29 & 4 & & 24 & 3 & .77 \\
HSGPA & 3.6 & .4 & & 3.5 & .5 & .01 \\
\hline
\end{tabular}

Note-Wilks's Lambda $=.92, p<.001 . \quad$ *Correlations between variables and canonical discriminant functions.

information. However, having the mathematical solution plus a three-dimensional display of the data that they can manipulate facilitates this interpretation. In Figure 1, the axes have been rotated so that the shape of the ellipsoid swarm is easily observed and the length of the major axis is maximized. In Figure 2, the HSGPA axis is perpendicular to the page, and the highly correlated relationship between the first factor and SAT and ACT is apparent. Similarly, in Figure 3, the axes have been rotated so that the SAT axis is perpendicular to the page and the other relationships can be examined. In this figure, the fact that HSGPA is not correlated with each of the other two measures or with the original variable is obvious. The ability to rotate the axes on which the data are plotted is an invaluable tool in the interpretation of factor analyses.

\section{Discriminant Analysis}

The single-factor multilevel MANOVA is the multivariate equivalent to the single-factor multilevel ANOVA. Similarly, Hotelling's $T^{2}(1951)$ is the multivariate equivalent to the univariate $F$ ratio and $t$ test. A one-way MANOVA (discriminant analysis) is applicable in cases in which there are two or more groups of subjects with more than one measure being obtained on each subject. The test of the overall null hypothesis is accomplished by reducing the set of $p$ measures to a single number by applying a linear weighting combination rule. The criteria for the application of the weighting rule is to maximize the difference between the groups. This results in the development of a set of weights (and factor loadings) that give the largest possible $F$ value for the difference in groups. This is not the traditional univariate $F$ ratio.

With the discriminant analysis, as with the factor analysis, a series of numeric values are used to draw conclusions and make inferences. In a discriminant analysis, the means and standard deviations of the $p$ variables, the factor loadings, the product-moment correlations, the weights, and the test of significance are used in interpreting the results. A great deal of the information contained in the numeric results describes the geometric relationship between the groups on the variables.

Figure 4 is a three-dimensional graphic of a two-group three-variable discriminant analysis. The null hypothesis is that there is no significant difference between these two groups on these three variables. The three variables are the ones used in the factor analytic example (SAT, ACT, and HSGPA), and the groups of subjects are males and females. The numeric results of the discriminant analysis are contained in Table 1.

It is apparent from the information contained in Table 1 that there is significant $(p<.01)$ overall difference between the two groups and that the null hypothesis is

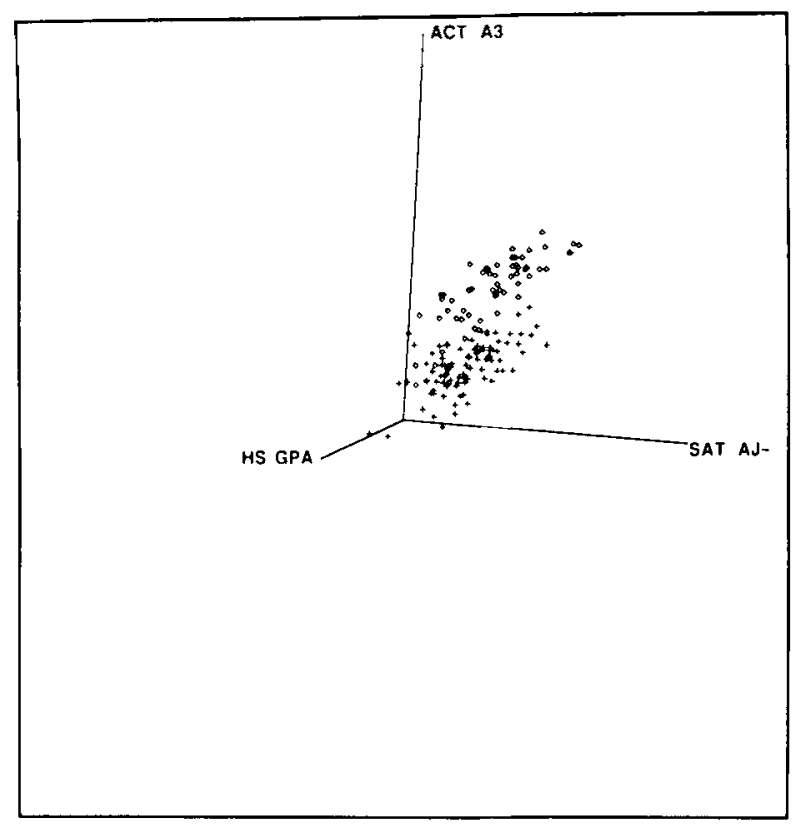

Figure 4. The same set of scores presented in Figure 1, but tested by discriminant analysis to show the difference between males' and females' scores. The axes have been rotated to show maximum difierence between groups ( $9^{\circ}$ latitude and $16^{\circ}$ longitude).

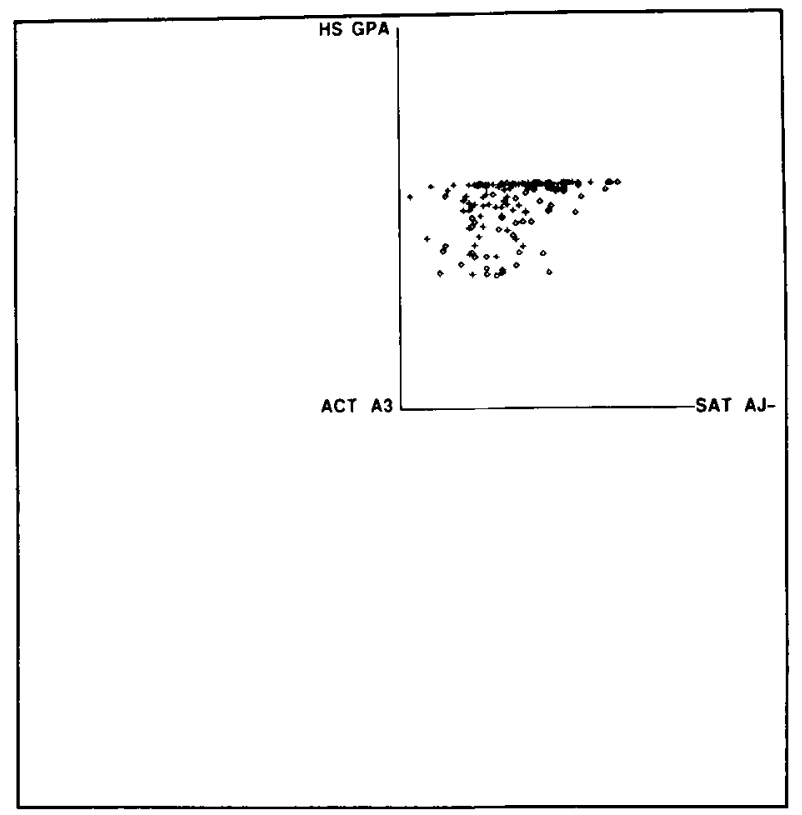

Figure 5. The same set of scores presented in Figure 1, but tested by discriminant analysis to show the difference between males' and females' scores. Viewed from the end of the ACT axes. Note that the test scores by gender are not discriminable from this perspective. 


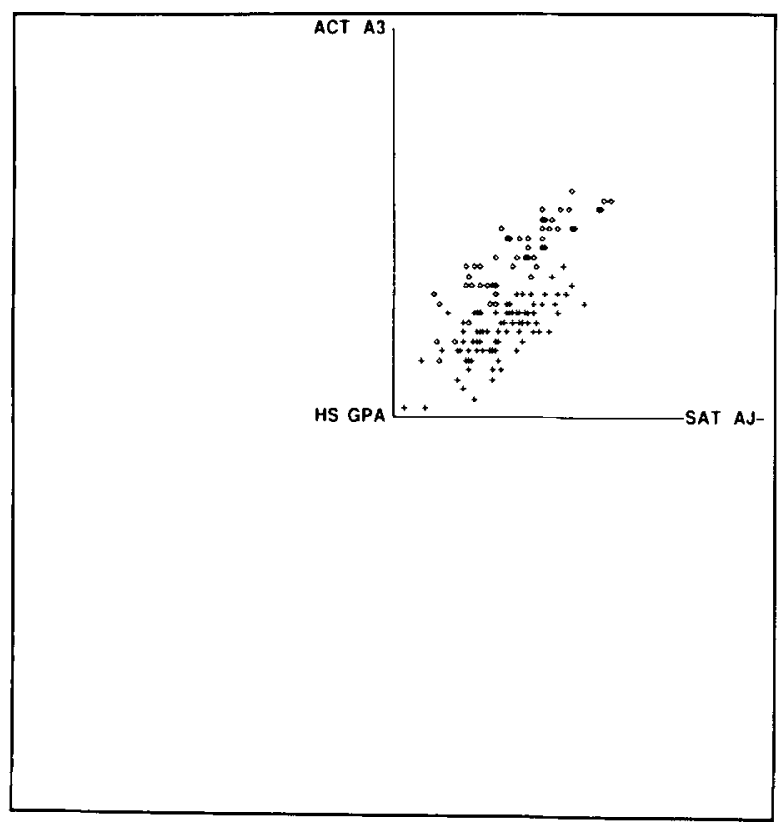

Figure 6. The same scores as in Figure 5, except that in this figure they are viewed from the end of the HS GPA axis. Note that the correlation between scores and the difference between scores by gender is evident.

rejected. The factor loadings indicate that SAT makes the largest contribution to the difference, that ACT also contributes to the difference, and that HSGPA makes almost no contribution to the difference. The classification table indicates that there is a $70 \%$ correct classification rate.

These results can be seen very clearly in Figure 4. When a set of weights is developed to give maximal discrimination between groups (males are represented by diamonds and females by pluses), the distance between the centroids (the geometric centers of the two threedimensional swarms-multivariate means) is being maximized. The separation or lack of overlap between groups is easily seen. Once this distance is established, a line connecting these two points and passing through the $x, y, z$ intercept becomes the first discriminant function. Using these three-dimensional graphics, one can find the first discriminant function by rotating the axes until the difference is maximized. Once this point is located, then there are several other pieces of information that become obvious. First, an investigator can see the shape or form of the multivariate distributions. That is, he/she can see the tightness or looseness of the distribution and the symmetry (or lack of symmetry) of the distribution. The MacSpin program permits a user to identify the names of subjects whose data are outlyers. Also, the amount of overlap in the distributions and the relative contribution of each of the variables to the overlap can be seen. The axes of Figure 4 have been rotated so that the discriminant function lies on the surface of the page. Note that the differences, overlap, and contributions to overlap can be seen in this figure.
In Figure 5, the axes have been rotated so that the ACT variable projects out of the page, and in Figure 6 they have been rotated so that HSGPA projects out of the page. This is equivalent to removing ACT (Figure 5) and HSGPA (Figure 6) from the analyses. Since there is no significant difference between groups on HSGPA, there is more overlap between the two groups then there was in Figure 4. Also note that when the effect of one variable (ACT) is removed, the "topping out" on HSGPA becomes obvious. It is also interesting to note that more females "topped out," or had a HSGPA of 4.0 , than did their male classmates. In Figure 6, the difference between the groups on the two achievement variables again becomes obvious. Note that the males tended to outscore the females on these two measures. It appears that the males tended to outscore the females on the two standardized achievement measures, whereas the females outscored the males on high-school grades.

A discriminant function in discriminant analysis is a direct parallel to a principal component in factor analysis. Similarly, there can be multiple orthogonal discriminant functions in a discriminant analysis, as there are multiple principal components in factor analysis. In factor analysis principal components explain the variance of correlated, composited variables, whereas in discriminant analysis the discriminant functions are used in discriminating between variables.

\section{Conclusions}

Many years ago, when we began to develop threedimensional graphics to use in conjunction with multivariate statistics, our objective was to develop systems that we could use as teaching tools. Advances in software and computer hardware development have permitted us to develop a usable system that exceeds our original expectations. The system is a good tutorial device, we are pleased with it, and our students seem to profit from its use. However, we believe that we have profited more from the use of the system than have the students. It is impossible to realize the value of three-dimensional graphics techniques until an investigator can sit down at a microcomputer and work directly with his/her own multivariate data.

\section{REFERENCES}

CLYDE, D. (1969). Multivariate analysis of variance on large computers. Coconut Grove, FL: Clyde Computing.

Donoho, A. W., Donoho, D. L., \& Gasko, M. (1986). MacSpin [Computer program]. Chicago, IL: D Software.

FinN, J. (1965). Multivariance. Toronto: University of Toronto Press. Kendall, M. G. (1969). On the future of statistics: A second book. Journal of the Royal Statistical Society, A131, 182-192.

Hotellivg, H. (1951). A generalized $t$-test and measures of multivariate dispersion. Proceedings of the Second Berkeley Symposium on Mathematical Statistics and Probability (pp. 23-41). Berkeley: University of California Press.

Hughes, E. F., LARuE, R., \& Yost, M. (1969). Multivariate analysis of variance on small computers, Coconut Grove, FL: Clyde Computing.

SAS INSTITUTE. (1986). SAS users guide: Basics. New York: Author. SPSS. (1985). SPSS users guide. New York: McGraw-Hill. 